\title{
Assistência qualificada no pré-natal como prevenção da violência obstétrica: Revisão integrativa
}

\author{
Qualified care in prenatal as a prevention of obstetric violence: Integrative review \\ Asistencia prenatal cualificada como prevención de la violencia obstétrica: Revisión integrativa
}

Recebido: 23/06/2021 | Revisado: 29/06/2021 | Aceito: 30/06/2021 | Publicado: 13/07/2021

Ranna Gabriele Sampaio da Conceição

ORCID: https://orcid.org/0000-0003-3449-5462

Universidade Estadual do Sudoeste da Bahia, Brasil

E-mail: ranna_gabriele@hotmail.com

Aline Vieira Simões

ORCID: https://orcid.org/0000-0001-5465-4980 Universidade Estadual do Sudoeste da Bahia, Brasil E-mail: avsimoes@uesb.edu.br

Vanda Palmarella Rodrigues

ORCID: https://orcid.org/0000-0002-5689-5910 Universidade Estadual do Sudoeste na Bahia, Brasil

E-mail: vprodrigues@uesb.edu.br

Juliana Costa Machado

ORCID: https://orcid.org/0000-0002-2258-0718 Universidade Estadual do Sudoeste da Bahia, Brasil E-mail: Juliana.costa@uesb.edu.br

Júlia Maria Nascimento Penha

ORCID: https://orcid.org/0000-0002-2334-7504 Universidade Estadual do Sudoeste da Bahia, Brasil E-mail: juliapenha1@hotmail.com

Franciele Soares Balbinote

ORCID: https://orcid.org/0000-0002-2502-9892 Universidade Estadual do Sudoeste da Bahia, Brasil E-mail: fran_balbinote@hotmail.com

\begin{abstract}
Resumo
A violência obstétrica compreende as agressões sofridas pelas gestantes durante o pré-natal, parto ou puerpério praticada pelos profissionais de saúde. Este estudo teve como objetivo analisar como a assistência à saúde ofertada pelos profissionais de saúde durante o pré-natal pode contribuir para a prevenção da violência obstétrica. Trata-se de uma revisão integrativa da literatura, coletada nas bases de dados Literatura Latino-Americana e do Caribe em Ciências da Saúde (LILACS), Medical Literature Analysisand Retrieval Sistem Online (MEDLINE) e no portal de periódicos Scientific Electronic Library Online (SciELO) indexados na Biblioteca Virtual de Saúde, através dos descritores "violência obstétrica", "assistência à saúde" e "pré-natal", com o auxílio do operador boleano AND. Foram selecionadas 11 publicações que versavam sobre a temática, em que se destacaram o desconhecimento das mulheres acerca da violência obstétrica, bem como o despreparo dos profissionais, devido às lacunas durante a graduação. Portanto, salienta-se a necessidade de os profissionais ofertarem um atendimento de qualidade, de maneira humanizada e individualizada desde o pré-natal até o puerpério, com esclarecimento das dúvidas e dos direitos legais que são assegurados por lei. Assim como, o desenvolvimento de capacitações para manejo e identificação da violência obstétrica para os profissionais e realização de atividades educativas para as gestantes, acompanhantes, entre outros.
\end{abstract}

Palavras-chave: Violência obstétrica; Assistência à saúde; Pré-natal; Saúde da mulher; Prevenção.

\begin{abstract}
Obstetric violence comprises the aggressions suffered by pregnant women during prenatal care, childbirth or puerperium practiced by health professionals. This study aimed to analyze how the health care offered by health professionals during prenatal care can contribute to the prevention of obstetric violence. This is an integrative literature review, collected in the Latin American and Caribbean Literature in Health Sciences (LILACS), Medical Literature Analysis and Retrieval System Online (MEDLINE) databases and the Scientific Electronic Library Online (SciELO) journal portal ) indexed in the Virtual Health Library, through the descriptors "obstetric violence", "health care" and "prenatal care", with the help of the Boolean operator AND. Eleven publications were selected that dealt with the theme, in which women's lack of knowledge about obstetric violence was highlighted, as well as the professionals' lack of preparation, due to gaps during graduation. Therefore, it emphasizes the need for professionals to offer quality care, in a humanized and individualized manner, from prenatal care to the puerperium, with
\end{abstract}


clarification of doubts and legal rights that are guaranteed by law. As well as the development of training for the management and identification of obstetric violence for professionals and educational activities for pregnant women, companions, among others.

Keywords: Obstetric violence; Health care; Prenatal care; Women's health; Prevention.

\section{Resumen}

La violencia obstétrica comprende las agresiones que sufren las gestantes durante la atención prenatal, parto o puerperio practicadas por profesionales de la salud. Este estudio tuvo como objetivo analizar cómo la atención de salud que ofrecen los profesionales de la salud durante la atención prenatal puede contribuir a la prevención de la violencia obstétrica. Se trata de una revisión integradora de la literatura, recopilada en las bases de datos de Literatura Latinoamericana y del Caribe en Ciencias de la Salud (LILACS), Sistema de Recuperación y Análisis de Literatura Médica en Línea (MEDLINE) y el portal de revistas Scientific Electronic Library Online (SciELO) indexadas en el Virtual Health Biblioteca, utilizando los descriptores "violencia obstétrica", "atención médica” y "atención prenatal", con la ayuda del operador booleano AND. Se seleccionaron once publicaciones que abordaron la temática, en las que se destacó el desconocimiento de las mujeres sobre la violencia obstétrica, así como la falta de preparación de los profesionales, por brechas durante la graduación. Por ello, enfatiza la necesidad de que los profesionales brinden una atención de calidad, de manera humanizada e individualizada, desde la atención prenatal hasta el puerperio, con el esclarecimiento de dudas y derechos legales que están garantizados por la ley. Así como el desarrollo de capacitaciones para el manejo e identificación de la violencia obstétrica para profesionales y actividades educativas para gestantes, acompañantes, entre otros.

Palabras clave: Violencia obstétrica; Cuidado de la salud; Prenatal; La salud de la mujer; Prevención.

\section{Introdução}

A Violência obstétrica é caracterizada por toda ação inerente e praticada pelos profissionais de saúde, no que se refere a respeito aos procedimentos reprodutivos das mulheres, que violem os seus corpos e acarretam traumas psicológicos. Podem se apresentar desde a gestação, no parto e no pós-parto, decorrente de uma assistência sem escuta qualificada, assim como não explanar e solicitar autorização para a realização de procedimentos, a utilização de palavras ofensivas ou o ato de impedir a autonomia da mulher, visto que a mesma é a protagonista durante e depois do parto fisiológico (Ismael et al, 2020).

No que tange ao Brasil, a violência obstétrica tem sido uma temática bastante abordada e que acomete muitas mulheres, seja no período pré-parto, pós-parto e abortamento, durante a assistência à saúde. As manifestações desse fenômeno vão desde agressões verbais, abuso de poder e perda de autonomia da parturiente, que refletem de forma negativa na vida dessas mulheres (Diniz et al, 2015).

Nesse sentido, a Organização Mundial de Saúde (OMS) ao considerar a violência obstétrica como um problema de saúde pública, emitiu a declaração: "Prevenção e eliminação de abusos, desrespeito e maus-tratos durante o parto em instituições de saúde", a qual visa assegurar o atendimento adequado e de qualidade, com acesso universal aos cuidados em saúde sexual e reprodutiva, trazendo visibilidade para prevenção e incentivo de intervenções das instituições públicas e privadas que prestam assistência às mulheres (OMS, 2014).

A violência obstétrica é subdividida em várias formas física, institucional, moral, sexual, psicológica e verbal; e apresentam implicações nocivas, como a flagelação de seus corpos, perda da autonomia e liberdade de escolhas de via de parto e plano de parto. Trata-se da violação de direitos a curto e a longo prazo, tais como: falta de informação, de autonomia e adesão de intervenções, além de práticas que são mutiladoras e prejudiciais, sem autorização da parturiente, que se destacam a episiotomia de rotina e a manobra de Kristeller. Estas são usadas sem restrições, alegando uma necessidade, sendo que, na maioria das vezes, o único objetivo é acelerar o parto (Brandt et al, 2018).

De acordo aos dados coletados pela Fundação Perseu Abramo, em uma pesquisa realizada em 2010, 25\% das mulheres brasileiras sofreu algum tipo de violência durante o parto, o que permite afirmar que uma em cada quatro mulheres vivenciou violência no momento do parto ou pré-natal, sendo mais frequente o exame de toque de maneira dolorosa e a negação de fármacos analgésicos. Para 23\% dessas mulheres pesquisadas, durante o parto ouviram algum despropósito. 
O poder executivo por meio dos representantes no Senado Federal e na Câmara dos deputados, recentemente, demonstrou resistência em utilizar a terminologia violência obstétrica e em três de maio de 2019, o Ministério da Saúde (MS) emitiu um documento que considerava a utilização da terminologia inadequada e que não agrega valor, questionando o fato de que os profissionais da saúde não a acometem, visando prejudicar ou causar danos ao binômio mãe-bebê, colocando em pauta a existência de programas e políticas de saúde que englobam a temática em questão (Brasil 2019a). Porém, o Ministério Público Federal de São Paulo (MPF) entrou com uma ação de recomendação para que o Ministério da Saúde reconhecesse e legitimasse o uso do termo. Assim sendo, no dia sete de junho de 2019, o Ministério da Saúde emitiu um documento oficializando o direito legítimo das mulheres e utilização do termo, bem como a utilização nas pesquisas, estudos e produções científicas sobre a violência obstétrica e que explanam diversas vertentes (Brasil, 2019b,2019c, Silva et al, 2021).

Outras discussões foram fomentadas sobre a institucionalização do parto a partir do Projeto de lei no 435/2019 na Câmera dos Deputados de São Paulo, tendo como autora a deputada Janaína Paschoal, que visa "garantir à gestante a possibilidade de optar pelo parto cesariano, a partir da trigésima nona semana de gestação, bem como a analgesia, mesmo quando escolhido o parto normal”. Posteriormente, esse projeto foi sancionado como a Lei nº 17.137/2019 (São Paulo, 2019, p.1).

Existe um outro Projeto de Lei $n^{\circ} 3.947$, de 2019, que está em tramitação no Senado Federal, tendo como autor o senador Sérgio Petecão, que em alguns trechos relata que: "[...] ficando as mulheres submetidas à verdadeira tortura, uma vez que não querem passar pelas dores e pelos riscos de um parto normal, mas não lhes é dada opção. Ademais, como já dito, surpreende saber que até mesmo a analgesia lhes é negada" (Brasil, 2019d, p.11).

A tramitação dessas leis denota a importância de discutir essa temática, bem como da divulgação e propagação das informações acerca da violência obstétrica para que a população tome conhecimento e que, principalmente as mulheres, tenham autonomia sobre as suas decisões e que cada vez mais se empoderem, além de evidenciar que os profissionais adotem outras posturas, com uma visão holística e que seja centralizada nos anseios das mulheres.

Assim, diante do exposto, foi elaborada a seguinte questão norteadora: De que forma uma assistência qualificada e adequada durante o pré-natal contribui para a prevenção da violência obstétrica?

Para responder esse questionamento, realizou-se este estudo com o objetivo de analisar como a assistência à saúde ofertada pelos profissionais de saúde durante o pré-natal pode contribuir para a prevenção da violência obstétrica.

\section{Metodologia}

Trata-se de um estudo de revisão integrativa de literatura que têm como objetivo proporcionar uma síntese de conhecimento a partir da aplicabilidade de resultados e estudos significativos na prática. A partir da definição do problema específico, determina o conhecimento mais atual por meio da identificação, análise e síntese dos dados provenientes dos estudos independentes sobre a mesma temática e, consequentemente, contribui para a disseminação das informações de forma positiva, tendo em vista a realidade observada (Souza, Silva \& Carvalho, 2010).

Para atender ao objetivo deste estudo, foi realizada uma busca na literatura utilizando as seguintes bases de dados: Literatura Latino Americana e do Caribe em Ciências da Saúde (LILACS), Medical Literature Analysis and Retrieval Sistem online (MEDLINE), e no portal de periódicos Scientific Electronic Library Online (SciELO), indexados na Biblioteca Virtual da Saúde (BVS), utilizando-se os descritores: "violência obstétrica", "assistência à saúde" e "pré-natal" e o operador boleano AND.

Inicialmente foi elaborada a seguinte questão norteadora: De que forma uma assistência qualificada e adequada durante o pré-natal contribui para a prevenção da violência obstétrica? 
Os critérios de inclusão adotados para seleção dos artigos foram: artigos publicados nos idiomas: português, inglês e espanhol; disponíveis na íntegra, que compreendessem o período de 2010 a 2020. Como critérios de exclusão: foram retirados artigos repetidos, que não se adequassem à temática e não completassem a questão norteadora, além de monografias, dissertações, teses ou outro tipo de documento.

A coleta foi realizada no período de junho a agosto de 2020, sob a perspectiva da Prática Baseada em Evidências (PBE) consiste numa metodologia utilizada para identificar a evidência e qualidade os estudos e mecanismos para implementação da assistência. A evidência científica representa a comprovação de que a temática é verdadeira ou falsa, por isso denota a importância da pesquisa prévia. A PBE é realizada, através das seguintes etapas: identificação de um problema clínico; formulação de uma questão crítica, busca de evidências científicas, avaliação, análise da aplicabilidade das evidências, implementação e conclusão dos resultados (Santos, Pimenta \& Nobre, 2007).

Buscou-se classificar o nível de evidência dos artigos considerando os seguintes pontos: 1. Revisões sistemáticas ou metanálise de relevantes ensaios clínicos; 2. Ao menos um ensaio clínico randomizado controlado bem delineado; 3. Ensaios clínicos bem delineados sem randomização; 4 . Estudos de coorte e de caso-controle bem delineados; 5 . Revisão sistemática de estudos descritivos e qualitativos; 6. Único estudo descritivo ou qualitativo; 7. Opinião de autoridades e/ou relatório de comitês de especialistas, além da interpretação de informações que não se basearam em pesquisas (Melnyk \& Fineout-Overholt, 2011).

Inicialmente foram encontrados 53 artigos. Foi feita uma leitura flutuante, para analisar a pertinência do material e após a utilização dos critérios de elegibilidade foram excluídos 33 artigos. Ademais, partiu-se para leitura na íntegra de 20 artigos, onde foram excluídos 9 artigos e utilizados 11 artigos para essa revisão integrativa.

Após a leitura, organização e discussão dos artigos selecionados, os resultados foram organizados em duas categorias temáticas: 1) Violência Obstétrica e suas repercussões e 2) Mecanismos de Prevenção da Violência Obstétrica.

\section{Resultados e Discussão}

Neste estudo foram utilizados 11 artigos científicos selecionados através dos critérios de inclusão estabelecidos anteriormente, sendo que a maioria apresentou nível de evidência 6, pois eram pesquisas qualitativas e os demais em nível de evidência 5, foram encontrados na Biblioteca Virtual de Saúde (BVS), conforme apresentado no Quadro 1.

Quadro 1. Artigos selecionados nas bases de dados BVS e PubMed.

\begin{tabular}{|c|c|c|c|c|c|}
\hline Título dos artigos & Autores & $\begin{array}{l}\text { Periódico } \\
\text { (vol, n, pag, } \\
\text { ano) }\end{array}$ & $\begin{array}{l}\text { Tipo de } \\
\text { estudo }\end{array}$ & $\begin{array}{l}\text { Nível de } \\
\text { evidência }\end{array}$ & Considerações/Temática \\
\hline $\begin{array}{l}\text { Mulher e parto: } \\
\text { significados da } \\
\text { violência obstétrica e } \\
\text { a abordagem de } \\
\text { enfermagem }\end{array}$ & $\begin{array}{l}\text { Oliveira, Elias } \\
\text { Oliveira }^{1}\end{array}$ & $\begin{array}{l}\text { Rev enferm } \\
\text { UFPE on line. } \\
\text { 2020;14:e243 } \\
996\end{array}$ & $\begin{array}{l}\text { Estudo } \\
\text { qualitativo }\end{array}$ & 6 & $\begin{array}{l}\text { Estudo aponta a necessidade do } \\
\text { fortalecimento da consulta do pré-natal } \\
\text { proporcionada pelo enfermeiro. }\end{array}$ \\
\hline $\begin{array}{l}\text { O saber de puérperas } \\
\text { sobre violência } \\
\text { obstétrica }\end{array}$ & Silva et al. ${ }^{2}$ & $\begin{array}{l}\text { Rev enferm } \\
\text { UFPE on line. } \\
\text { 2019;13:e242 } \\
100\end{array}$ & $\begin{array}{l}\text { Estudo } \\
\text { qualitativo }\end{array}$ & 6 & $\begin{array}{l}\text { Estudo ressalta a importância do } \\
\text { conhecimento das puérperas acerca da } \\
\text { violência obstétrica para poderem } \\
\text { identificar e intervir, caso a prática ocorra. }\end{array}$ \\
\hline $\begin{array}{l}\text { Violência Obstétrica } \\
\text { Institucional no } \\
\text { Parto: Percepção de } \\
\text { Profissionais da } \\
\text { Saúde }\end{array}$ & $\begin{array}{l}\text { Cardoso, Costa, } \\
\text { Almeida, Santos } \\
\& \text { Oliveira }^{3}\end{array}$ & $\begin{array}{l}\text { Rev enferm } \\
\text { UFPE on } \\
\text { line., Recife, } \\
\text { 11(9):3346- } \\
\text { 53, set., } 2017\end{array}$ & $\begin{array}{l}\text { Estudo } \\
\text { qualitativo }\end{array}$ & 6 & $\begin{array}{l}\text { Estudo aponta que a grande maioria dos } \\
\text { profissionais desconhecem a violência } \\
\text { obstétrica }\end{array}$ \\
\hline
\end{tabular}




\begin{tabular}{|c|c|c|c|c|c|}
\hline $\begin{array}{l}\text { Fatores associados à } \\
\text { satisfação do } \\
\text { acompanhante com o } \\
\text { cuidado prestado à } \\
\text { parturiente }\end{array}$ & Batista et al. ${ }^{4}$ & $\begin{array}{l}\text { Cogitare } \\
\text { enfermag; } \\
\text { 22(3): } 01-09 \\
\text { jul-set. } 2017 \text {. }\end{array}$ & $\begin{array}{l}\text { Estudo } \\
\text { transversa } \\
1\end{array}$ & 5 & $\begin{array}{l}\text { Avalia a satisfação do acompanhante com } \\
\text { o cuidado prestado à mulher durante o } \\
\text { trabalho de parto. }\end{array}$ \\
\hline $\begin{array}{l}\text { Elaboração de Novas } \\
\text { Tecnologias em } \\
\text { Enfermagem: } \\
\text { Utilização de Uma } \\
\text { Cartilha de } \\
\text { Prevenção }\end{array}$ & Santos, Souza ${ }^{5}$ & $\begin{array}{l}\text { Rev enferm } \\
\text { UFPE on } \\
\text { line., Recife, } \\
\text { 11(10):3893- } \\
8, \text { out., } 2017\end{array}$ & $\begin{array}{l}\text { Estudo } \\
\text { Qualitativ } \\
\text { o }\end{array}$ & 6 & $\begin{array}{l}\text { O artigo demonstra a importância de } \\
\text { tecnologias interativas no processo de } \\
\text { cuidado. }\end{array}$ \\
\hline $\begin{array}{l}\text { À margem da } \\
\text { humanização? } \\
\text { Experiências de parto } \\
\text { de usuárias de uma } \\
\text { maternidade pública } \\
\text { de Porto Alegre-RS }\end{array}$ & $\begin{array}{l}\text { Pedroso, López } \\
\text { Pand }\end{array}$ & $\begin{array}{l}\text { Physis (Rio } \\
\text { J.); 27(4): } \\
\text { 1163-1184, } \\
\text { Out.-Dez. } \\
2017 .\end{array}$ & $\begin{array}{l}\text { Estudo } \\
\text { qualitativo }\end{array}$ & 6 & $\begin{array}{l}\text { O artigo relata a escolha da maternidade } \\
\text { como decisão crítica, as visões sobre o } \\
\text { parto e a violência obstétrica. }\end{array}$ \\
\hline $\begin{array}{l}\text { Obstetrizes e } \\
\text { enfermeiras obstetras } \\
\text { no Sistema Único de } \\
\text { Saúde e na Atenção } \\
\text { Primária à Saúde: por } \\
\text { uma incorporação } \\
\text { sistêmica e } \\
\text { progressiva }\end{array}$ & Norma, Tesser ${ }^{7}$ & $\begin{array}{l}\text { Revista } \\
\text { brasileira de } \\
\text { medicina de } \\
\text { família e } \\
\text { comunidade; } \\
\text { 10(34): } 1-7, \\
\text { jan./mar. } \\
2015 \text {. }\end{array}$ & $\begin{array}{l}\text { Estudo } \\
\text { qualitativo }\end{array}$ & 6 & $\begin{array}{l}\text { O artigo apresenta proposta de } \\
\text { incorporação de obstetrizes e enfermeiras } \\
\text { obstetras na Atenção Primária à Saúde, } \\
\text { visando à redução da violência obstétrica. }\end{array}$ \\
\hline $\begin{array}{l}\text { Violência obstétrica: } \\
\text { perfil assistencial de } \\
\text { uma maternidade } \\
\text { escola do interior do } \\
\text { estado de São Paulo }\end{array}$ & $\begin{array}{l}\text { Biscegl, Grio, } \\
\text { Melles, } \\
\text { Ribeiro \& } \\
\text { Gonsaga }^{8}\end{array}$ & $\begin{array}{l}\text { CuidArte, } \\
\text { Enfermagem; } \\
9(1): 18-25, \\
\text { jan.-jun.2015. }\end{array}$ & $\begin{array}{l}\text { Estudo } \\
\text { transversa } \\
1\end{array}$ & 5 & $\begin{array}{l}\text { Descreve as características do atendimento } \\
\text { de uma maternidade-escola e verifica a } \\
\text { existência da violência obstétrica na } \\
\text { mesma. }\end{array}$ \\
\hline $\begin{array}{l}\text { Violência obstétrica } \\
\text { como questão para a } \\
\text { saúde pública no } \\
\text { Brasil: origens, } \\
\text { definições, tipologia, } \\
\text { impactos sobre a } \\
\text { saúde materna, e } \\
\text { propostas para sua } \\
\text { prevenção. }\end{array}$ & Diniz et al. ${ }^{9}$ & $\begin{array}{l}\text { Revista } \\
\text { brasileira } \\
\text { crescimento e } \\
\text { desenvolvime } \\
\text { nto humano; } \\
\text { 25(3): } 377- \\
384,2015 \text {. }\end{array}$ & $\begin{array}{l}\text { Revisão } \\
\text { crítico- } \\
\text { narrativa }\end{array}$ & 5 & $\begin{array}{l}\text { Define, tipifica, cita os impactos e formas } \\
\text { de prevenção para a violência obstétrica. }\end{array}$ \\
\hline $\begin{array}{l}\text { A peregrinação no } \\
\text { período reprodutivo: } \\
\text { uma violência no } \\
\text { campo obstétrico }\end{array}$ & $\begin{array}{l}\text { Rodrigues et } \\
\text { al. }{ }^{10}\end{array}$ & $\begin{array}{l}\text { Escola Anna } \\
\text { Nery Rev. } \\
\text { Enferm; } \\
\text { 19(4): 614- } \\
\text { 620, out.-dez. } \\
\text { 2015. }\end{array}$ & $\begin{array}{l}\text { Estudo } \\
\text { qualitativo }\end{array}$ & 6 & $\begin{array}{l}\text { Aborda a peregrinação em busca de } \\
\text { atendimento obstétrico como forma de } \\
\text { violência obstétrica e os sentimentos } \\
\text { ocasionados pelo fato. }\end{array}$ \\
\hline $\begin{array}{l}\text { Obstetric Violence } \\
\text { and Its Associated } \\
\text { Factors Among } \\
\text { Postnatal Women in a } \\
\text { Specialized } \\
\text { Comprehensive } \\
\text { Hospital, Amhara } \\
\text { Region, Northwest } \\
\text { Ethiopia }\end{array}$ & $\begin{array}{l}\text { Mihret, } \\
\text { Muhabaw } \\
\text { Shumye }^{11}\end{array}$ & $\begin{array}{l}\text { BMC. Res } \\
\text { Notes. } \\
\text { 2019;12(1):60 } \\
0\end{array}$ & $\begin{array}{l}\text { Estudo } \\
\text { transversa } \\
1 .\end{array}$ & 5 & $\begin{array}{l}\text { Estudo aponta alta prevalência de violência } \\
\text { obstétrica em hospital da Etiópia e os } \\
\text { fatores envolvidos. }\end{array}$ \\
\hline
\end{tabular}

Fonte: Dados da pesquisa.

Foram analisados quatro artigos do ano 2017, quatro artigos do ano 2015, dois artigos do ano 2019 e um artigo do ano 2020. Dos artigos selecionados, a maior parte trata-se de pesquisa qualitativa, sendo sete artigos dessa modalidade; três estudos transversais e uma revisão crítico narrativa. 
Destaca-se a lacuna de artigos específicos sobre a importância de uma assistência qualificada durante o pré-natal, entretanto no que se refere à temática da violência obstétrica nota-se uma maior facilidade nos achados.

Após a análise crítica dos artigos selecionados, foi realizada a leitura dos resumos disponíveis em cada artigo, buscando verificar se os documentos encontrados condiziam com a pergunta de investigação. Os dados foram distribuídos e organizados de acordo com a relação da ideia principal dos mesmos e os resultados foram organizados em duas categorias temáticas que serão apresentadas abaixo.

\section{1) Violência Obstétrica e suas Repercussões}

Os estudos agrupados nessa temática versam sobre a tipologia e as diversas faces da violência obstétrica, a qual se constitui uma prática muito frequente e recorrente nas instituições de saúde, sendo perpetrada pelos profissionais de saúde no âmbito de suas atividades, tornando esse tipo violência numa forma institucionalizada, reducionista e mecânica, em que as mulheres são colocadas em segundo plano (Cardoso et al, 2017; Pedroso \& López, 2017).

A violência obstétrica constitui um grave problema de saúde pública que acomete diversas mulheres numa escala mundial, sendo relatada inicialmente a partir de 1950, através das maneiras arcaicas e desatualizadas que os profissionais de saúde exercem no atendimento e acompanhamento das parturientes trazem consequências negativas, além de infligir e ferir autonomia, a dignidade e o direito dessas mulheres (Diniz et al, 2015).

Estudos apontaram como os profissionais agem de maneira mecânica, seja durante o pré-natal, parto e pós-parto, em que as mulheres se tornam um número e mais um procedimento, com intervenções desnecessárias, muitas vezes sem autorizações e sem explicações. Essas condutas podem acarretar em danos físicos e psicológicos, podendo ser irreversíveis, num momento em que a mulher deveria ser protagonista, mas se torna coadjuvante e sem voz, tendo seus anseios e desejos desconsiderados. Outro fator importante que os artigos abordam, diz respeito ao fato de os profissionais terem uma postura conservadora e desatualizada inerente apenas às práticas cirúrgicas e mecânicas, e que a humanização se perdeu ao longo do caminho (Pedroso \& López, 2017; Biscegli et al, 2015).

Ademais afirmaram o desconhecimento das parturientes quanto à violência obstétrica, visto que durante o pré-natal as informações e orientações sobre a temática não são explicadas, fazendo com que essas mulheres não percebam que foram violadas e violentadas durante o parto. Justamente esse, que seria um momento para ser natural/fisiológico, torna-se patológico, com intervenções desnecessárias como a episiotomia de rotina, administração de medicamentos, amniotomia, violência verbal, entre outras formas de violência obstétrica que acontecem (Oliveira, Elias \& Oliveira, 2020; Silva et al, 2019; Cardoso et al, 2017).

O despreparo, a negligência e a atenção desumanizada são os fatores preponderantes e que remetem à hierarquização e hegemonia dos profissionais da saúde, principalmente no que se diz respeito à relação médico-paciente que inferiorizam as mulheres e banalizam a violência obstétrica com a finalidade de acelerar os processos e procedimentos. Destaca-se que as mulheres mais acometidas, são as pardas, multíparas, sem instrução e que, pelo fato de não terem o conhecimento sobre a temática, acreditam que as medidas adotadas são normais e necessárias para que tudo ocorra bem e não cause danos ao bebê. Ressalta-se também a necessidade das discussões sobre a temática em questão durante a graduação, bem como um aprofundamento, tendo como base evidências científicas atualizadas (Pedroso \& López, 2017; Biscegli et al, 2015; Diniz et al, 2015; Mihret, 2019).

\section{2) Mecanismos de Prevenção da Violência Obstétrica}

Os estudos coadunam sobre a importância de uma assistência humanizada e holística, que priorize a autonomia, a individualidade e o protagonismo dessas mulheres, para a redução da morbimortalidade e prevenção da violência obstétrica, 
bem como a adoção de ações educativas, desde o pré-natal, além de possibilitar a tomada de consciência das mulheres sobre seus direitos e sobre a capacidade de reconhecerem as faces da violência obstétrica (Oliveira, Elias \& Oliveira, 2020; Silva et al, 2019; Pedro \& López, 2017; Norman \& Tesser, 2015).

Algumas políticas públicas vigentes são eficazes, se fiscalizadas, como por exemplo, a Lei do Acompanhante de 2005, que assegura à parturiente o direito de escolha de um acompanhante durante o pré-natal, parto e o pós-parto imediato, frisando que a presença do pai, fortalece o trinômio mãe-pai-filho e reduz os índices de violência obstétrica. Existe ainda a Lei $\mathrm{n}^{\circ} 11.634 / 2007$, que institui a visita da gestante na maternidade de referência, para conhecimento e vinculação, onde receberá assistência no âmbito do Sistema Único de Saúde (SUS). Outra estratégia que vem sendo utilizada é a produção e distribuição de cartilhas educativas, com orientações sobre os direitos da mulher, as fases da gestação e como funcionam o pré-natal, o parto, o pós-parto e o papel que os profissionais de saúde desempenham nos atendimentos (Brasil, 2005, 2007; Batista et al, 2017; Santos \& Souza, 2017; Norman \& Tesser, 2015; Rodrigues et al, 2015, Silva et al, 2021).

Pesquisa realizada em parceria entre o Brasil e o Reino Unido, demonstra que a influência inglesa entre as enfermeiras e obstetrizes, que realizam desde o pré-natal de baixo risco e o pós-parto imediato, acarreta na diminuição das taxas de cesáreas e na redução dos índices da violência obstétrica. O que denota a importância da inserção desses profissionais no âmbito da Atenção Primária à Saúde (APS), para fortalecimento de uma assistência de qualidade e humanizada, o que requer maiores investimentos nas políticas públicas em saúde da mulher e que os gestores se predispõem a terem a iniciativa de forma eficiente e eficaz (Norman \& Tesser, 2015).

Foram apontadas como as formas da violência obstétrica, seja: física, verbal e/ou psicológica, imposição de intervenções não consentida, entre outros, podem se apresentar e se desenvolver durante o acompanhamento dessas mulheres, assim como, relatam maneiras e mecanismos para prevenção, redução e extinção desse tipo violência, tendo como base uma boa preparação acadêmica, com uma formação holística e humanizada, fundamentada em evidências científicas atualizadas, que fomentem e contribuam para um atendimento de qualidade, principalmente na APS. Ademais, apontam sobre a relevância da participação e implementação dessas ações pelas enfermeiras obstétricas e obstetrizes, na oferta da assistência de excelência e que faça a diferença na vida dessas mulheres, orientando e esclarecendo as dúvidas e reiterando o empoderamento feminino, tornando-a protagonista desse momento sublime, que é o processo de partejar (Batista $e t$ al, 2017; Santos \& Souza, 2017; Norman \& Tesser, 2015; Diniz et al, 2015).

\section{Conclusão}

O estudo demonstrou que a violência obstétrica está atrelada diretamente à assistência desumanizada, desatualizada, sem uso de evidências científicas pertinentes e embasadas. Para reduzi-la e extingui-la, se faz necessária uma abordagem aprofundada, através de cursos e/ou capacitações dos profissionais de saúde, de forma que as parturientes recebam um atendimento adequado e holístico.

Foi possível perceber que existe uma lacuna no processo de formação desses profissionais, pois o assunto em questão não é visto com frequência, sendo evidenciada numa residência ou numa pós-graduação, que necessitam de capacitações para manejo e identificação da violência obstétrica, posto que a grande maioria demonstra o despreparo e desconhecimento.

Os estudos também apontam a carência de melhorias e atualizações nas políticas públicas da saúde da mulher, no fortalecimento de ações educativas para conscientização e prevenção, favorecendo o protagonismo e empoderamento dessas mulheres e permitindo que ocorra uma articulação entre os serviços que resultem na redução da morbimortalidade e nos danos decorrentes da prática irrestrita da violência obstétrica.

Sendo assim, conclui-se que as mulheres devem receber um atendimento de qualidade, de maneira humanizada e individualizada desde o pré-natal até o puerpério, com esclarecimento das dúvidas e dos direitos que lhe são assegurados por 
lei. Nesse quesito, os profissionais de enfermagem têm papel fundamental no desenvolvimento de discussões sobre a temática durante as consultas de pré-natal, não apenas às mulheres, mas sempre que possível ao acompanhante que também deve participar das consultas, assim como no desenvolvimento de rodas de conversas, cursos e cartilhas para melhor orientarem e prestarem um atendimento de qualidade.

Ademais, diante da relevância do tema, espera-se o desenvolvimento de outros estudos que abordem sobre a inserção da temática durante a graduação no intuito de preparar os futuros profissionais para uma assistência humanizada, bem como incentivar o empoderamento das mulheres para manutenção do seu protagonismo e de propagar o conhecimento sobre a violência obstétrica para toda população, auxiliando no combate a esse fenômeno devastador.

\section{Referências}

Batista, B. D. et al. Fatores associados à satisfação do acompanhante com o cuidado prestado à parturiente. (2017). Cogitare Enfermagem. 22(3).

Biscegli, T. S., Grio, J. M., Melles, L. C., Ribeiro, S. R. M. I., \& Gonsaga, R. A. T. (2015) Violência obstétrica: perfil assistencial de uma maternidade escola do interior do Estado de São Paulo. Revista cuidarte enfermagem. 9(1),18-25.

Brandt, G. P., Souza, S. J. P., Migoto, M. T., \& Weigertl, S. P. (2018). Violência obstétrica: a verdadeira dor do parto. Revista Gestão \& Saúde. 9(1), 19-37.

Brasil (2007). Diário Oficial da União - Lei no 11.634, de 27 de dezembro de 2007. <http://www.planalto.gov.br/ccivil_03/_Ato2007-2010/2007/Lei/L11634 .htm>.

Brasil (2005). Diário Oficial da União - Lei $n^{o} 11.108$, de 07 de abril de 2005. <http://www.planalto.gov.br/ccivil_03/_ato2004-2006/2005/lei/111108.htm>.

Brasil (2019a). Ministério da Saúde. Secretaria de Atenção à Saúde. Departamento de Ações Programáticas Estratégicas. Despacho DAPES/SAS/MS, Brasília, 03 de maio de 2019. < https://sei.saude.gov.br/sei/controlador_externo.php?acao=documento_conferir\&codigo verific ador=9087621\&codigo_c rc=1A6 F34C4\&hash_do wnload=c4c55cd95ede706d0b729845a5d6481d07e735f33d87d40984dd1b39a32d870fe89dcf1014bc76a32d2a28d8f0a2c5ab928ff16 5c67d8 219 e3 5beb1a0adb3258\&visualizacao=1\&id_orgao_acesso_externo=0>.

Brasil (2019b). Ministério Público Federal. Recomendação no 29/2029, de 07 de maio de 2019, Ministério Público Federal de São Paulo recomenda que o Ministério da Saúde se abstenha de empregar quaisquer ações voltadas especificamente à abolição do uso da expressão "violência obstétrica". <http://www.mpf.mp.br/sp/sala-de-imprensa/docs/recomendacao_ms_violencia_obstetrica.pdf/>.

Brasil. (2019c). Ministério da Saúde. Departamento de Ações Programáticas Estratégicas. Coordenação de ciclos de Vida. Coordenação de Saúde das Mulheres, Ofício No 296/2019, de 07 de junho de 2019. <http://www.mpf.mp.br/sp/sala-de-imprensa/docs/oficio-ms.>

Brasil (2019d). Projeto de Lei $n^{\circ} 3.947$, de 2019. Senado Federal. <https://www25.senado.leg.br/web/atividade/materias/-/materia/137641>.

Cardoso, F.J. C., Costa, A. C A. M., Almeida, M. M., Santos, T. S., \& Oliveira, F. B. M. (2017). Violência obstétrica institucional no parto: percepção dos profissionais de saúde. Revista de Enfermagem UFPE. 11(9), 3346-3353.

Diniz, S. G., Salgado H. de O., Andrezzo, H. F. de. A., Carvalho, P. G. C., Carvalho, P. C. A., Aguiar, C. de. A., \& Niy, D. Y. (2015). Violência obstétrica como questão para a saúde pública no brasil: origens, definições, tipologia, impactos sobre a saúde materna, e propostas para sua prevenção. Periódicos Eletrônicos em Psicologia, São Paulo. 25(3), 377-384.

Ismael, F. M., Souza, G. K. R., Esteves, N. S., \& Aoyama, E. A. (2020). Assistência de Enfermagem na Prevenção da Violência Obstétrica. Revista Brasileira Interdisciplinar de Saúde, Distrito Federal. 2(2), 75-80.

Fundação Perseu Abramo \& Sesc. (2010). Pesquisa de opinião pública: mulheres brasileiras e gênero nos espaços público e privado.

Melnyk, B. M., \& Fineout-Overholt, E. (2011) Making the case for evidence-based practice. In: MELNYK, B.M; FINEOUT-OVERHOLT, E. Evidence-based practice in nursing \& healthcare: a guide to best practice [Internet]. $2^{\mathrm{a}}$ ed. Philadelphia: Lippincot Williams \& Wilkins. 3-24.

Mihret, M. S. (2019). Obstetric violence and its associated factors among postnatal womwn in a specialized comprehensive hospital, Amhara region, Northwest Ethiopia.Violência obstétrica e seus fatores associados entre mulheres pós-parto em um Hospital Abrangente Especializado, Região de Amhara, Noroeste da Etiópia. BMC Res Notes. 12(1), 600.

Norman, A. H., \& Tesser, C. D. (2015). Obstetrizes e enfermeiras obstetras no Sistema Único de Saúde e na Atenção Primária à Saúde: por uma incorporação sistêmica e progressiva. Rev Bras Med Fam Comunidade. 10(34),1-7.

Oliveira, M. R. R., Elias, E. A., \& Oliveira, S. R. (2020). Mulher e parto: significados da violência obstétrica e a abordagem de enfermagem. Rev enferm UFPE on line. $14: \mathrm{e} 243996$.

Pedroso, C. N. L da S., \& López, L. C. (2017). À margem da humanização? Experiências de parto de usuárias de uma maternidade pública de Porto AlegreRS. Physis: Revista de Saúde Coletiva. 27(4), 1163-1184.

Rodrigues, D. P., Alves, V. H., Penna, L. H. G., Pereira, A.V., Branco, M. B. L. R., \& Silva, L. A. (2015). A peregrinação no período reprodutivo: uma violência no campo obstétrico. Esc. Anna Nery, Rio de Janeiro, 19(4), 614-620. 
Research, Society and Development, v. 10, n. 8, e34910817505, 2021

(CC BY 4.0) | ISSN 2525-3409 | DOI: http://dx.doi.org/10.33448/rsd-v10i8.17505

Santos, A. L. M., \& Souza, M. H. T. (2017). Elaboração de novas tecnologias em enfermagem: utilização de uma cartilha de prevenção. Revista de Enfermagem UFPE on line. 11(10), 3893-3898.

Santos, C. M. da C., Pimenta, C. A. de M., \& Nobre, M. R. C. (2007). A estratégia pico para a construção da pergunta de pesquisa e busca de evidências. Rev Latino-am de Enfermagem.15(3),508-511.

São Paulo (Estado). Lei $n^{\circ} 17.137$, de 23 de agosto de 2019. Garante à parturiente a possibilidade de optar pela cesariana, a partir de 39 (trinta e nove) semanas de gestação, bem como a analgesia, mesmo quando escolhido o parto normal.

Silva, F. C., Viana, M. R. P., Amorim, F. C. M., Veras, J. M. M. F., Santos, R. C., \& Sousa, L. L. (2019). O saber de puérperas sobre violência obstétrica. Rev enferm UFPE on line. 13:e242100.

Silva, A. F. P.M., Almeida, B. G. R. F., Ribeiro, E. A., Teixeira, L. C., Silva, P. C. P. O., \& Ribeiro, A. da S. (2021). Violência obstétrica relacionada a perda de autonomia da mulher na sala de parto. Research, Society and Development. 10(5), e22210514814.

Souza, M. T., Silva, M. D., \& Carvalho, R. (2010). Revisão integrativa: o que é e como fazer. Einstein. 8(1), 102-106. 